\title{
Mucoadhesive Microspheres of Atorvastatin Calcium: Rational Design, Evaluation and Enhancement of Bioavailability
}

\author{
Hitesh Kumar Dewangan', Arpana Sharma², Ankit Mishra², Pradeep Singour ${ }^{2, *}$ \\ ${ }^{1}$ Institute of Pharmaceutical Research (IPR), GLA University, Mathura, Uttar Pradesh, INDIA. \\ ${ }^{2}$ Faculty of Pharmacy, VNS Group of Institution, Bhopal, Madhya Pradesh, INDIA.
}

\begin{abstract}
Background: Atorvastatin Calcium (ATC) used for lowering the cholesterol levels in body. It is competitive inhibitor of hydroxyl methylglutaryl-coenzyme A (HMG-CoA) reductase, followed mevalonate pathway, which have low bioavailability and poor solubility. Present work focus on development of mucoadhesive microspheres of atorvastatin calcium, for improve the delayed transit, continuous longer period release and preclinical pharmacokinetic estimation in rabbit. Materials and Methods: Microsphere was prepared by emulsification method in which the independent variables (like polymer amount) were studied on critical quality attributes like entrapment efficiency, percentage yield and in-vitro release. Microspheres were characterized in terms of physicochemical parameters, micromeritic properties, FT-IR, DSC and mucoadhesive wash-off test and further, evaluated for their pharmacokinetics study in rabbits. Results: The designed microsphere exhibited an average size with smooth surface, negative zeta potential, maximum entrapment efficiency and sustained release. Microspheres fulfil the micromeritic properties and showed no any interaction between drug and polymer, confirmed by FT-IR and DSC. The in-vivo study demonstrated that the prepared microspheres are effective for colon targeted drug delivery system at longer duration. In pharmacokinetics study, relatively steady plasma drug concentrations were observed within after oral administration of drug. The $\mathrm{AUC}_{0-24 \mathrm{~h}}$ for formulation were significantly higher than that of pure drug $(p<0.05)$. Conclusion: The pre-clinical oral bioavailability study of drug was increased as the relative availability values were high compared with pure drug and it showed delayed transit for longer period of time.
\end{abstract}

Key words: Atorvastatin calcium, Mucoadhesive, Microspheres, Colon targeting, Bioavailability.

\section{INTRODUCTION}

Atorvastatin Calcium [(3R,5R)-7-[2( 4 - flu or ophen y 1 ) - 3 - phe n y 1 4-(phenylcarbamoyl)-5-propan-2-ylpyrrop1yl]-3,5-dihydroxyheptanoic acid [ATC] is a member of the drug used for lowering the cholesterol levels in body. The primary uses of atorvastatin calcium are for the treatment of disease like dyslipidemia and the prevention of cardiovascular disease. ${ }^{1-3}$ It is competitive inhibitor of hydroxyl methylglutaryl-coenzyme A (HMG-CoA) reductase, followed mevalonate pathway.
The rate-determining enzyme in cholesterol biosynthesis via the mevalonate pathway. ${ }^{4,5}$ In mevalonate pathway HMG-CoA convert to mevalonate in presence of catalyses HMG-CoA reductase. ATC is primarily act on liver cells. In which decrease the hepatic and plasma cholesterol levels. ${ }^{6,7}$

ATC showed low bioavailability and poor solubility. The rate of absorption of ATC was maximum found in the upper GI tract. ${ }^{8} 9$ Therefore, mucoadhesive drug system are utilize for the enhancement of
Submission Date: 30-11-2020; Revision Date: 03-03-2021; Accepted Date: 09-08-2021

DOI: 10.5530/ijper.55.3s.180 Correspondence:

Dr. Pradeep Singour, Faculty of Pharmacy VNS Group of Institution, Bhopal-462044, Madhya Pradesh, INDIA

Phone: +91 8934916022, Email-psingour18@gmail. com

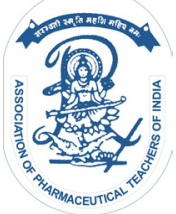

www.ijper.org 
bioavailability. Mucoadhesive drug system provides the targeted controlled and sustained release of ATC for longer period of time. ${ }^{10}$ It is based on delayed transit and continuous release system, so it lives in prolong residence in GIT especially in colon, along with their release. ${ }^{11}$ The colon is a site for local and systemic delivery of drugs. Treatment can be made by directly target into colon, so that enhancement of bioavailability and reduction of systemic side effects. ${ }^{12,13}$

The most acceptable oral administration mucoadhesive system of drug offers patient convenience, less pain, less risk of cross infection and injuries caused by needle stick. The rationale is to, a colon delivery system is very specific and quite diverse. The drug substance direct absorbed at the site of colon due to a long transit time, present of neutral $\mathrm{pH}$, proffer of reduced digestive enzymes and responsiveness of absorption enhancers. Thus, the targeting is reliable, effective and completed. Another is to lower surface area and relative 'tightness' of the tight junction in the colon, this can also restricted for transportation of drug across the mucosa layer and into the systemic circulation. ${ }^{14,15}$

Now, present works represent the development and evaluation of mucoadhesive polymeric microspheres. These mucoadhesive polymeric microspheres having an intimate contact with the mucus layer, so improve absorption at the targeting site, enhance bioavailability of the drugs and retention in the gastrointestinal tract. In this paper, different formulations of polymeric microspheres were prepared and evaluated their physicochemical properties (like particle size, entrapment efficiency, percentage yield, morphology), drug release studies, micromeritic properties, FT-IR, thermogram study and mucoadhesive properties. Pharmacokinetics profile of developed microsphere was performed in rabbit.

\section{MATERIALS AND METHODS}

Atorvastatin Calcium purchased from Cipla Pharmaceutical Indore. Carbopol and sodium alginate were provided by Oxford chemicals laboratory, Mumbai. Light Liquid Paraffin, Calcium Chloride and Span 80 were obtained from Loba chemical laboratory, Mumbai. All other chemicals as well as Buffer salts used in experiments were analytical grade reagent. Animal used in experiment were treated according to the protocols and the studies were carried out as per the guidelines of CPCSEA (Council for the Purpose of Control and Supervision of Experiments on Animals), Government of India.

\section{Preparation and optimization of microspheres}

Fabrication of the drug loaded microspheres (DMPs) is represented in Table 1. In the optimization only one formulation parameter such as ratio of polymer was changed at a time while the remaining variables were kept constant. The amounts of cross-linking agents $(5 \%$ calcium chloride) and drug amount was kept constant and calculating the percentage drug entrapment and in-vitro drug release (\%). Microsphere was prepared by emulsification method, followed by cross-linking with calcium chloride. Firstly, Atorvastatin calcium was dispersed in aqueous solution of sodium alginate (5\%). Prepared this aqueous solution was again emulsified with light liquid paraffin containing span 80 using a homogenizer at $2000 \mathrm{rpm}$ for $120 \mathrm{~min}$. $5 \mathrm{ml}$ of $2 \%$ calcium chloride was added to the emulsion. The solidified microspheres were recovered by centrifugation, washed with petroleum ether and dried in vacuum desiccators. Formulation was observed in microscope under $100 \mathrm{x}$ magnifications. ${ }^{16,17}$

\section{Physicochemical characterization of microspheres}

The average particle size, polydispersity index and zeta potential of the microsphere were determined by Zeta sizer nano ZS90, Malvan UK analyser. Samples were prepared by after re-suspension of prepared formulation in distilled water. All measurements were performed in triplicates. ${ }^{18}$ The morphology of atorvastatin calcium loaded microspheres was carried out by a scanning electron microscope (Hitachi High Technology, Pleasanton, CA). Diluted samples was dropped into stubs and allow for air drying, then coated with gold (thin layer) and observed under scanning electron microscopy. ${ }^{19,20}$

The amount of ATC loading in the developed microsphere was quantified using direct method. Microspheres were extracted with methanol and dilution with PBS ( $\mathrm{pH}$ 6.8) in appropriate quantity and analysed by reverse phase high-performance liquid chromatography (Jasco, PU2080 pump HPLC) method containing $5 \mu \mathrm{m} \mathrm{C}_{18}$ column (Hi Qsil, $250 \mathrm{~mm} \times 0.45 \mathrm{ID}$ ) and 70:30 ratio of acetonitrile and phosphate buffer as a mobile phase, with a $1 \mathrm{ml}$ per minute flow rate at $247 \mathrm{~nm}$ wavelength. ${ }^{21}$

Drug Loading Efficiency $=\frac{\text { Weight of drug in microsphere }}{\text { Weight of the microsphere }} \times$ eig

Percentage yield $=\frac{\text { Weight of the dried microspheres obtained }}{\text { Total weight of drug and polymer used }} \times 100$

In-vitro release of atorvastatin calcium from microsphere was carried out by USP paddle type dissolution test apparatus (Model ZRS-8, China). Near about $10 \mathrm{mg}$ of 
atorvastatin Calcium loaded microsphere were placed in dissolution vessels and dissolution medium containing $900 \mathrm{ml}$ of phosphate buffered saline $(\mathrm{pH}$ 6.8) and maintained $37 \pm 0.5^{\circ} \mathrm{C}$ temperature at $50 \pm 5 \mathrm{rpm}$. At the predetermine time intervals an aliquot amount $(5 \mathrm{ml})$ of the dissolution media was withdrawn and same amount of fresh solvent was replaced immediately. Withdrawn samples were further proceed for reverse phase HPLC analysis at $247 \mathrm{~nm} .^{22}$ After that the release kinetic was studied by various kinetic models as zero order, first order, Higuchi plot and Korsemeyer-Peppas model. The best fit model was confirmed by the value of correlation coefficient near to one.

\section{Determination of micromeritic properties}

\section{Angle of Repose}

Microspheres were passed through a fixed funnel, which is stayed in specific height upon graph paper. Due to only gravity force a static heap of powder was found on graph paper. The height (h) and radius(r) of cone were measured. The angle of repose was calculated using formula. ${ }^{23}$

$\tan \theta=\mathrm{h} / \mathrm{r}$

Therefore

$$
\theta=\tan ^{-1} \mathrm{~h} / \mathrm{r}
$$

Where $\theta=$ Angle of repose, $h=$ height of cone and $r=$ radius of cone base.

\section{Carr's Index}

To check the flow property of prepared microsphere was conducted by comparing the poured and tapped density of a sample. Small amount of microsphere sample was taken in measuring cylinder. Samples height was measured before and after tapping, which represent the poured and tapped density respectively. ${ }^{24}$ Further, Carr's Index was calculated as:

$$
\mathrm{I}=\left(\mathrm{V}_{\mathrm{b}}-\mathrm{V}_{\mathrm{t}} / \mathrm{V}_{\mathrm{b}}\right)^{*} 100
$$

Where $\mathrm{V}_{\mathrm{b}}=$ bulk volume and $\mathrm{V}_{\mathrm{t}}=$ tapped volume

\section{Determination of Swelling Properties}

Swelling index measure the extend of swelling properties of particles in $6.8 \mathrm{pH}$ phosphate buffer. For this purpose weighted amount of $100 \mathrm{mg}$ microspheres were allowed to swell for $24 \mathrm{hr}$ in $6.8 \mathrm{pH}$ phosphate buffer. After $24 \mathrm{hr}$ excess liquid were removed by blotting paper and microspheres were weighed. The degree of swelling was then calculated by the following formula

$$
\text { Degree of swelling }=\left(M_{o}-M_{t} / M_{t}\right)^{* 100}
$$

Where $M_{t}=$ Microspheres initial weight and $M_{0}=$ Weight of microspheres at equilibrium swelling in the media. ${ }^{25}$

\section{FTIR spectroscopy study}

To verify the possible interaction between drug and polymer, flourier transform infrared (JASCO-FTIR, Model-8300) analysis was conducted. Samples of pure atorvastatin calcium, sodium alginate, carbopol 934, physical mixture and prepared formulation were scanned in range from $400-4000 \mathrm{~cm}^{-1}$. All samples were triturated with $\mathrm{KBr}$ to get a very fine powder. Powder was transferred to pellet forming die and force applied to get a thin pellet. Pellet was fixed in a die given in IR instrument and scanned. ${ }^{26,27}$

\section{Differential scanning calorimetry study}

The possibility of any interaction between drug and polymer was assessed by carrying out the thermal analysis of atorvastatin calcium, sodium alginate, carbopol 934, physical mixture and prepared formulation. The thermal behaviour was determined using differential scanning calorimeter (DSC Q10 V9.4 Build 287) at continue heating rate. The measurements were performed at a heating range of $0^{\circ} \mathrm{C}$ to $400^{\circ} \mathrm{C}^{28}$

\section{In-vitro mucoadhesion wash-off test}

Mucoadhesive property of microspheres was estimated by in-vitro adhesion test. Eggshell membrane is suitable technique for this purpose. In this $2 \times 1 \mathrm{~cm}$ piece of eggshell membrane were taken and fixed on a glass slide (at an angle of $45^{\circ} \mathrm{C}$ ). About $100 \mathrm{mg}$ microspheres were spread on rinsed, tissue specimen and hung onto one of the groves of a USP tablet disintegrating test apparatus containing $6.8 \mathrm{pH}$ phosphate buffer. The disintegrating test apparatus was started, the tissue specimen showed regular up and down movements in a beaker. The time required for detaching of microspheres from mucosal surface membrane was recorded by visual inspection. ${ }^{29,30}$

\section{Pharmacokinetic study of microspheres}

Albino rabbit (2-3 $\mathrm{Kg}$ Body weight) were used in the pharmacokinetic study. All the rabbits were housed in a cage and maintained on $12 \mathrm{hr}$ light/dark at room temperature $25^{\circ} \mathrm{C}$ with free access to water and pelleted diet. Animals were deprived of food for $24 \mathrm{hr}$ before experiment. Atorvastatin calcium $25 \mathrm{mg} / \mathrm{kg}$ dose was administered to group first and selected formulation equivalent to $25 \mathrm{mg}$ was given to group second. Blood samples $(3 \mathrm{ml})$ were collected in marginal ear vein at $30,60,120,180,300,420$ and $1440 \mathrm{~min}$. and further centrifuged at $10000 \mathrm{rpm}$ for $20 \mathrm{~min}$ to separate serum and stored at $-20^{\circ} \mathrm{C}$ until analysis. Estimation of drug 
was performed by reverse phase HPLC (Jasco, PU2080 pump HPLC) method containing $5 \mu \mathrm{m} \mathrm{C}_{18}$ column (Hi Qsil, 250mm x 0.45 ID) and 70:30 ratio of acetonitrile and phosphate buffer, with a $1 \mathrm{~mL}$ per minute flow rate at $247 \mathrm{~nm}$ wavelength. ${ }^{21}$ Further pharmacokinetic parameters were calculated like $\mathrm{C}_{\max }, \mathrm{T}_{\max }$ and AUC. AUC (Area under the plasma level-time curve) can be calculated by trapezoidal rule. The extent of bioavailability can be determined by following equation

$$
\operatorname{Fr}=\frac{[\text { AUC]test Dstd }}{[\text { AUC]std Dtest }}
$$

Where $\mathrm{Fr}=$ Relative availability

\section{Statistical Analysis}

The two-tailed $t$-test was applied between groups for measurement of statistical significance differences. The method involves dividing the curve by a series of vertical lines into a number of trapezoids, calculating separately each trapezoid and adding them together.

\section{RESULTS AND DISCUSSION}

\section{Optimization and physicochemical characterization}

The mucoadhesive microspheres were prepared by emulsification method followed by cross-linking with calcium chloride. Optimization of formulation involved selection of external phase, internal phase, dispersing agent, washing solvent and mainly depend upon the ratio of two polymer concentration. The comparatively studies of $\%$ drug entrapment, percentage yield and in-vitro drug release profile presented in Table 1. The percentage drug loading of microspheres varied from to $37.7 \pm 1.34 \%$ to $65.9 \pm 0.03 \%$. Results demonstrated that an increase in concentration of sodium alginate increased the percentage drug entrapment due to availability of active calcium binding sites in the polymeric chains and quantity of sodium alginate which gives greater degree of cross linking. ${ }^{31}$ The optimized $\mathrm{AH}_{4}$ formulation code selected because when the change in ratio of polymer (500:500), \% drug entrapment and in-vitro drug release fluctuated. ${ }^{18}$ The size and PDI of ATC loaded selected microsphere batch were found to be $3.82 \pm 0.07 \mu \mathrm{m}$ and $-30 \mathrm{mv}$. Microsphere exhibited good polydispersity and micron size range. Prepared microsphere formed a homogeneous population and fairly spherical in shape confirm by scanning electron microscope (Figure 1). The

\begin{tabular}{|c|c|c|c|c|c|c|}
\hline í & 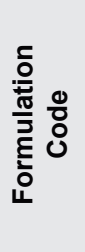 & 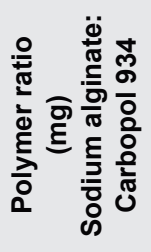 & 올 & 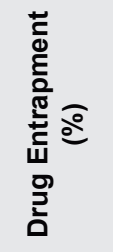 & $\begin{array}{l}\frac{0}{0} \\
\frac{\pi}{5} \\
\frac{0}{0} \\
\frac{0}{0} \\
\frac{0}{0}\end{array}$ & 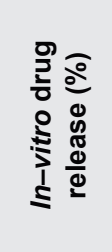 \\
\hline 1. & $\mathrm{AH}_{1}$ & 100: 900 & 100 & $\begin{array}{c}32.1 \pm \\
1.77\end{array}$ & $\begin{array}{l}37 \pm \\
0.32 \\
\end{array}$ & $\begin{array}{c}79.22 \pm \\
0.29\end{array}$ \\
\hline 2. & $\mathrm{AH}_{2}$ & 200: 800 & 100 & $\begin{array}{c}34.45 \pm \\
0.33 \\
\end{array}$ & $\begin{array}{l}44 \pm \\
0.45 \\
\end{array}$ & $\begin{array}{c}77.45 \pm \\
0.11 \\
\end{array}$ \\
\hline 3. & $\mathrm{AH}_{3}$ & 300: 700 & 100 & $\begin{array}{c}39.2 \pm \\
1.12 \\
\end{array}$ & $\begin{array}{l}49 \pm \\
0.25 \\
\end{array}$ & $\begin{array}{c}75.85 \pm \\
1.23 \\
\end{array}$ \\
\hline 4. & $\mathrm{AH}_{4}$ & 500: 500 & 100 & $\begin{array}{c}60.3 \pm \\
0.31\end{array}$ & $\begin{array}{c}60.2 \pm \\
0.51 \\
\end{array}$ & $\begin{array}{c}72.75 \pm \\
0.26\end{array}$ \\
\hline 5. & $\mathrm{AH}_{5}$ & 700: 300 & 100 & $\begin{array}{c}61.4 \pm \\
0.12\end{array}$ & $\begin{array}{c}63.1 \pm \\
0.75\end{array}$ & $\begin{array}{c}71.51 \pm \\
0.21\end{array}$ \\
\hline 6. & $\mathrm{AH}_{6}$ & 800: 200 & 100 & $\begin{array}{c}62.8 \pm \\
1.25\end{array}$ & $\begin{array}{c}65.2 \pm \\
1.3\end{array}$ & $\begin{array}{c}69.71 \pm \\
1.01\end{array}$ \\
\hline 7. & $\mathrm{AH}_{7}$ & 900: 100 & 100 & $\begin{array}{c}63.3 \pm \\
1.03\end{array}$ & $\begin{array}{c}68.2 \pm \\
1.1\end{array}$ & $\begin{array}{c}67.65 \pm \\
0.70\end{array}$ \\
\hline
\end{tabular}
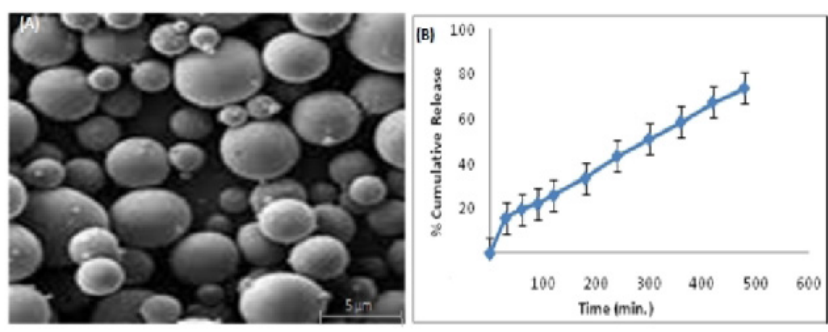

Figure 1: (A) Morphology of prepared microsphere by scanning electron microscope. (B) In-vitro drug release of optimized microsphere.

particles were also showed a smoothness. The selected optimized batch was found $60.3 \pm 0.31 \%$ drug entrapment and $37 \pm 0.32 \%$ yield.

The in-vitro drug release profiles of all formulations were studies in phosphate buffer solution at $\mathrm{pH} 6.8$. The commutative release of the drug from the polymeric microsphere shell of different formulation were shown in Figure 2. Release of drug is also depend upon the ratio of polymer. The $\mathrm{AH}_{1}$ showed maximum $\%$ cumulative release $(77.39 \pm 0.24 \%)$, which having the polymer ratio was $(100: 900)$ while $\mathrm{AH}_{7}$ showed minimum $66.08 \pm 0.20 \%$ cumulative release, which having the polymer ratio was $(900: 100)$. It was observed that, when increases the amount of sodium alginate, also the number of $\mathrm{COOH}$ groups is increased, which is further cross linked with $\mathrm{Ca}^{2+}$ ions and consequential in a formation of more intact matrix, which makes more difficulty of the drug release. The selected optimized 


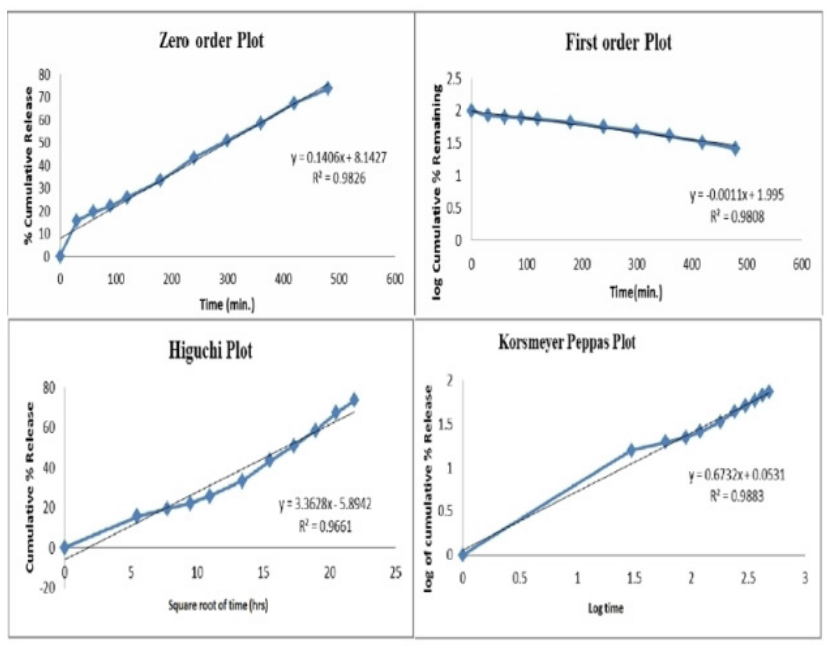

Figure 2: In vitro release kinetic of ATC loaded microsphere in phosphate buffer (pH 6.8) using different models such as: Zero order, First order, Higuchi model and KorsmeyerPeppas model.

batch was found to be $72.75 \pm 0.26 \%$ drug release. The microsphere exhibited sustained release of drug from polymeric shell because matrix degradation. Several all-other mechanisms like disintegration, desorption, diffusion, surface and bulk erosion were also involved (Figure 2). The microsphere showed an initial burst release; thus, the release of drug was very rapidly.

\section{Determination of micromeritic properties}

All formulation showed good flow except formulation $\mathrm{AH}_{7}$, because it contains more concentration of sodium alginate, having more cross linking between sodium alginate and calcium chloride. The swelling behaviour of both polymers were the major factor for controlling the release of the drug from the microspheres. The degree of swelling increases as increases the concentration of mucoadhesive polymer Carbopol 934. Figure 3 represent the relative swelling Index $\%$ of all the formulations.

\section{FTIR spectroscopy of drug and polymers}

FTIR spectroscopy checks the possible interaction between drug and polymer. It works on selective absorption of light by vibration modes of specific chemicals bonds. The observation of vibration spectrum of pure atorvastatin calcium, sodium alginate, carbopol 934, physical mixture and prepared formulation was found. There should be no any chemical interaction will be shown in Figure 4.

\section{Differential Scanning Calorimetry}

The DSC thermograms of plain drug, sodium alginate, polymer, physical mixture and microspheres are shown

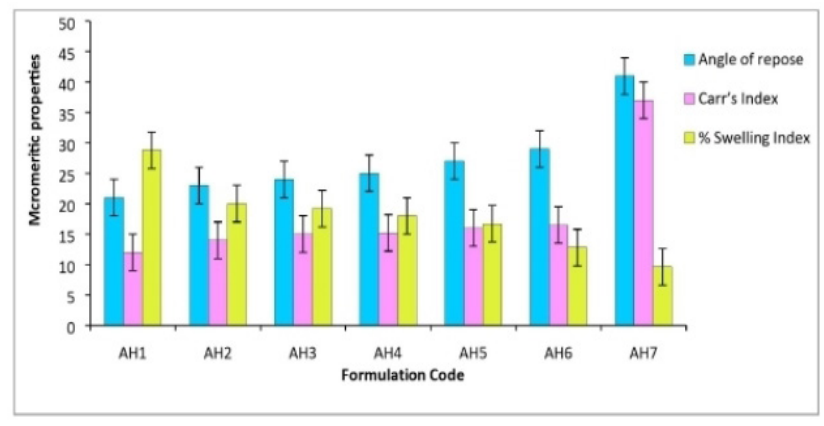

Figure 3: Comparatively studies of micromeritic properties like, Series 1: Angle of repose, Series 2: Carr's Index, Series 3: \% Swelling Index.

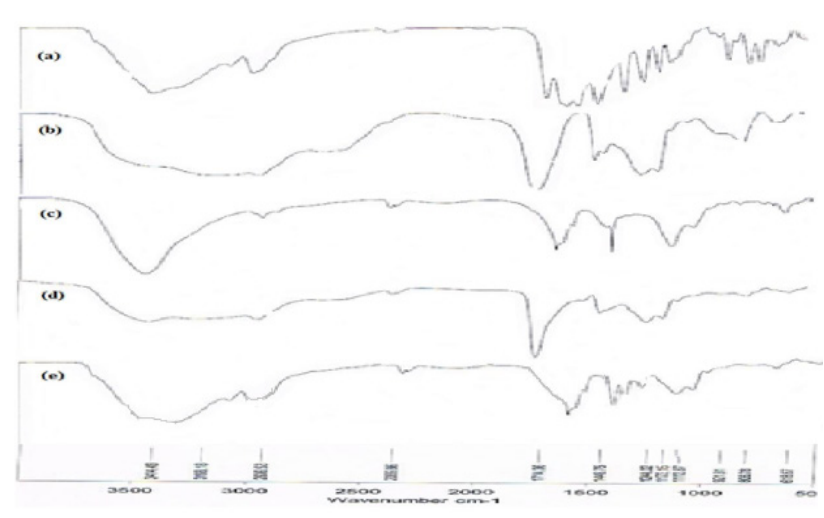

Figure 4: FTIR spectrum of (a) drug (b) Carbopol 934 (c) Sodium Alginate (d) physical mixture (e) microsphere formulation.

in Figure 5. The thermograms represent an endothermic peak of atorvastatin calcium, carbopol 934 and sodium alginate at $158^{\circ} \mathrm{C}$ to $178^{\circ} \mathrm{C}, 70$ to $80^{\circ} \mathrm{C}$ and $65^{\circ} \mathrm{C}$ to $70^{\circ} \mathrm{C}$ respectively. Characteristic peaks of atorvastatin calcium and sodium alginate were well recognized in the drugpolymer physical mixture. Results of DSC thermogram of microspheres indicate that, there are no any physical interaction between the drug and polymer when it was encapsulated in polymeric coat.

\section{In-vitro mucoadhesion wash-off test}

In-vitro adhesion testing method was utilized for the evaluation of mucoadhesive property of microspheres. In this study, carbopole have a quality to strong interaction with mucous. Presence of more amount of carbopol, greater retention was noticed. In order to evaluate the mucoadhesion time egg shell membrane was used. It is the substitute of animal stomach mucosa because of similarity (with respect to composition and thickness) between the eggshell membrane and the stomach mucous. Figure 6, shown relative mucoadhesion time of all the formulations. 


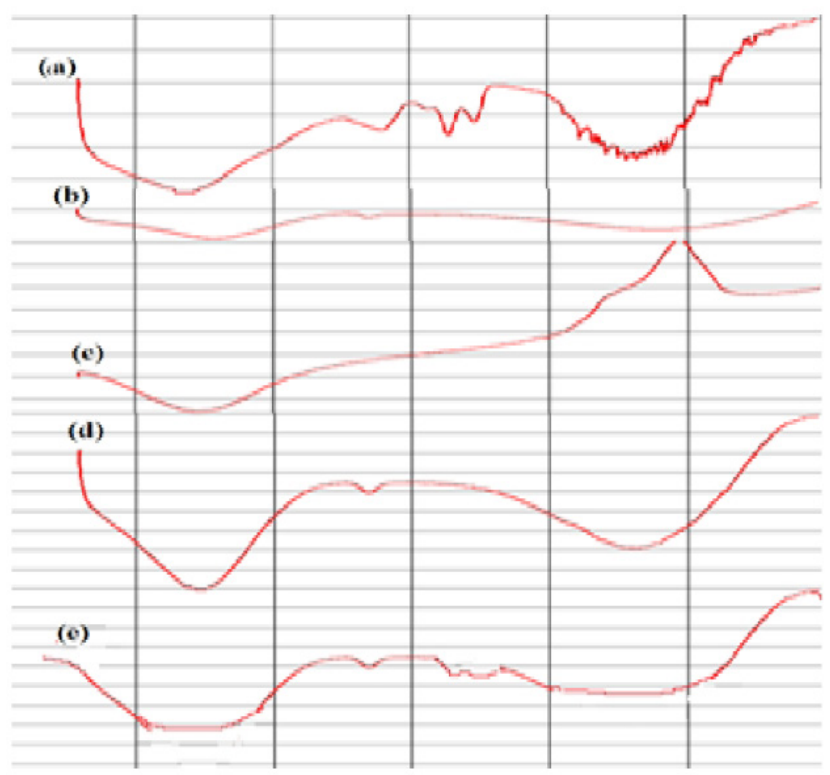

Figure 5: DSC spectrum of (a) drug (b) Carbopol 934 (c) Sodium Alginate (d) physical mixture (e) microsphere formulation.

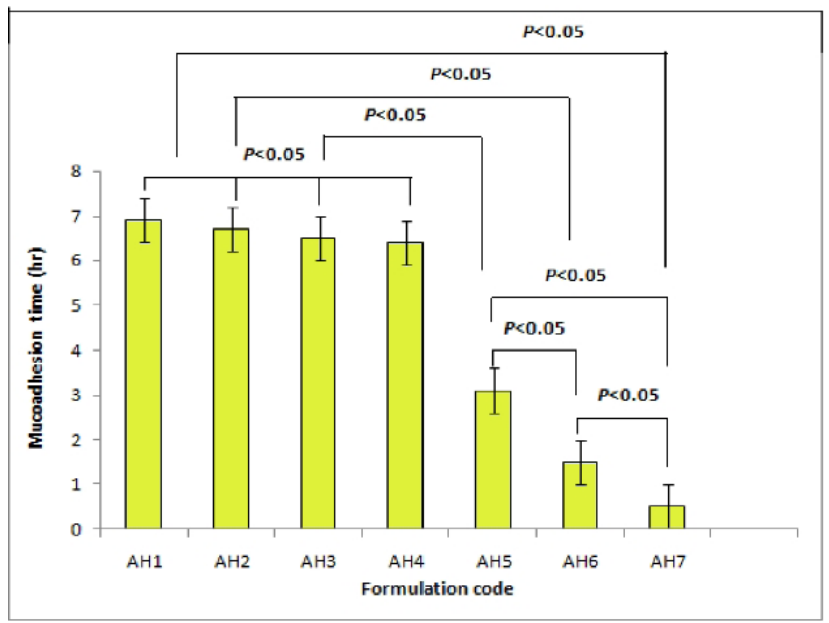

Figure 6: Relative mucoadhesion time of prepared different formulations.

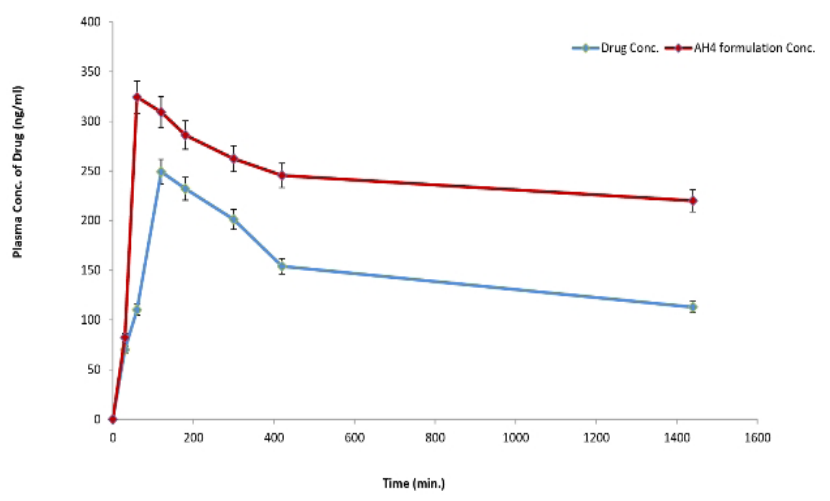

Figure 7: Comparatively graph of plasma concentration versus time profile of optimized formulation batch and drug.

\section{Pharmacokinetic study of microspheres}

The plasma concentration versus time profile of optimized formulation $\mathrm{AH}_{4}$ was shown in Figure 7. After oral administration of the drug, drug concentration reached at peak plasma concentration within $2 \mathrm{hr}$ and then rapidly decreased. The optimized formulation of $\mathrm{AH}_{4}$ reached rapidly at peak plasma concentration within $1 \mathrm{hr}$. and given maximum activity. Comparative peak plasma concentration of drug and $\mathrm{AH}_{4}$ formulation was shown, the $\mathrm{C}_{\max }$ values for drug and $\mathrm{AH}_{4}$ formulation were $249.2 \pm 0.77 \mathrm{ng} / \mathrm{ml}$ and $324.45 \pm$ $1.24 \mathrm{ng} / \mathrm{ml}$ respectively in $2 \mathrm{hrs}$ and $1 \mathrm{hr}$ with $\mathrm{T}_{\max }$ values. The $\mathrm{AUC}_{0-24 \mathrm{~h}}$ for drug and $\mathrm{AH}_{4}$ formulation was $3541.49 \pm 22.01 \mathrm{ng} . \mathrm{h} / \mathrm{mL}$ and $5095.40 \pm 13.77 \mathrm{ng} . \mathrm{h} / \mathrm{mL}$. The results shown $\mathrm{C}_{\max }$ values and $\mathrm{AUC}_{0-24 \mathrm{~h}}$ for $\mathrm{AH}_{4}$ formulation were significantly higher than that of drug $(p<0.05)$. Therefore, the oral relative bioavailability of drug was increased (43\%) compared with pure drug. All results were considered statistically significant when $(p<0.05)$, unless otherwise specified. ${ }^{29}$

\section{DISCUSSION}

The atorvastatin calcium loaded microsphere has been successfully developed to improvement of the bioavailability. After the selection of polymer and excipients, applied an emulsification method for development of microsphere. As the optimization, amount of polymer was directly affecting the microsphere entrapment efficiency and size. Prepared microsphere was characterized their physicochemical properties, such as particle size, zeta potential, entrapment efficiency and morphology. The morphology of microsphere showed smoothness and fairly spherical in shape. The physicochemical properties of atorvastatin calcium are having very slightly soluble in aqueous phase environment so the systemic availability is low and attributed to presystemic clearance in gastrointestinal mucosa or hepatic first-pass metabolism. The in vitro release of atorvastatin calcium showing the maximum amount of drug should be released in polymeric matrix system. In encapsulated or matrix system the drug release is depend upon the amount of encapsulated drug and the polymeric initial burst effect. Generally, when the amount of drug will be higher in encapsulated particle, the release of drug will be also higher. Secondly the particle size affects the dissolution or the release of ATC microsphere. In the gastrointestinal tracts smallest size of particles is easily absorbed though paracellularly followed passive diffusion and reach in systemic circulation. 
In a formulation of atorvastatin calcium drug release is also depend upon the ratio of polymer. It was observed that, when increase in the amount of one polymer sodium alginate increases, the number of $\mathrm{COOH}$ groups which were cross linked by $\mathrm{Ca}^{2+}$ ions resulting in a formation of more intact matrix which makes the drug release more difficult. The maximum release and loading formulation will be achieved. The in-vitro drug release studies data were plotted in various kinetic models, (zero order, first order, Higuchi models and Korsemeyer Peppas models), to determine the actual drug release process. The interpretation of data was based on the value of the resulting regression coefficients. Microsphere showed an initial burst release therefore release of drug which adsorbed on to the surface of the microsphere was rapid. It also demonstrated sustained release of ATC from polymeric shell due to matrix degradation and followed other release mechanisms like bulk erosion, disintegration, diffusion and desorption. Prepared microspheres were free from any possible interaction with excipients, which is confirmed by FTIR and DSC studies.

Further, several studies have demonstrated regarding the atorvstatin calcium, in the gastrointestinal tracts ATC absorbed though paracellularly followed passive diffusion. Mucoadhesive microsphere formulation is one of the most capable and continuous drug release approaches which could be retained the drug in the colon. Previous study reported that the gastrointestinal transit time is more if the various mucoadhesive materials incorporated into microspheres. Mucoadhesive materials like carbopol is widely used materials, it having a carboxyl groups which formed the bonds between hydrogen and mucus. The activity of the carbopol is affected by mainly two factors like ionic strength and $\mathrm{pH}$ of mucosa. Another is presence of sodium alginate which added in microspheres is an attempt to retard the drug release. For the mucoadhesion evaluation of microsphere, a newly developed eggshell membranes were utilized which is substitute of animal mucosa. Now, the microsphere was prepared and evaluated, to the targeting of colon by delayed transit and continuous sustained release of microspheric particles. After oral administration of the drug pharmacokinetic of the atorvastatin calcium having the plasma peak concentrations are lower (approximately $30 \%$ for $\mathrm{C}_{\text {max }}$ and AUC) follow by evening drug administration compared with morning. However, LDL-C reduction is the same regardless of the time of day of drug administration while the formulated microsphere particles reached rapidly at highest peak plasma concentration. In comparison to pure drug, $\mathrm{C}_{\max }$ and $\mathrm{AUC}_{0-24 \mathrm{~h}}$ value were significantly higher. Moreover, the prepared microsphere is suitable and effective for improvement of bioavailability due to prolonged gastric residence.

\section{CONCLUSION}

The prepared mucoadhesive microspheres which is composed of carbopol 934 and sodium alginate cross linking, having a good approach to improve the gastric residence time and bioavailability of drug due to mucoadhesive nature of polymer carbopol 934. Sodium alginate was added in microspheres is an attempt to retard the drug release. After administration of atorvastatin calcium loaded microspheres in rabbits, the bioavailability of drug increased as compared with that of pure drug. The pharmacokinetic study like bioavailability was increased in rabbits due to extensive gastric residence time.

\section{ACKNOWLEDGEMENT}

All authors are thankful to institute for given that the necessary facilities. I would like thank to Central Instrument Facility, IIT (Banaras Hindu University) for characterization of synthesized compounds.

\section{CONFLICT OF INTEREST}

The authors declare no conflict of interest.

\section{ABBREVIATIONS}

ATC: Atorvastatin Calcium; FTIR: Flourier Transform Infrared; DSC: Differential Scanning Calorimetry; HPLC: High Performance Liquid Chromatography.

\section{REFERENCES}

1. Ahjel SW, Lupuleasa D. Enhancement of solubility and dissolution rate of different form of atorvastatin calcium in direct compression tablet formulas. Farmacia. 2009;57(3):290-300.

2. Sharma B, Saini V, Sharma A. Preparation characterization and in-vitro evaluation of atorvastatin calcium solid dispersions with various hydrophilic polymers and its FDT formulation. Current Pharma Research; 2012;2(4). p. 620-30.

3. Igea SA, Espinar FJO, Vilajato JL, et al. The properties of solid dispersions of clofibrate in polyethylene glycols. Int J Pharm. 1995;70:57-66.

4. Eroglu H, Nemutlu E, Turkoglu OF, Nacar O, Bodur E, Sargon MF, Beskonakli E, Oner L. A Quadruped Study on Chitosan Microspheres Containing Atorvastatin Calcium: Preparation, Characterization, Quantification and $<i>$ in-Vivo</i> Application. Chem Pharm Bull. 2010;58(9):1161-7. doi: 10.1248/cpb.58.1161.

5. Aramă CC, Drăghici I, Monciu CM, et al. Assay of some hypocholesterolemiant substances (lovastatin, simvastatin) using NP-HPLC. Farmacia. 2007;LV(3):267-73.

6. Drăghici I, Nedelcu A, Monciu CM, et al. Derivative spectrometry in the assay of simvastatin and lovastatin. Farmacia. 2008;LVI(1):5-14. 
7. Indian Pharmacopoeia, government of India Ministry of Health and Family Welfare, Vol. I. Delhi: controller of publication, 143-4.

8. Tripathi KD. Essential of medical pharmacology. New Delhi: Jaypee Brothers medical publishers. PMID 2008612-621.

9. Kadu PJ, Kushare SS, Thacker DD, Gattani SG. Enhancement of oral bioavailability of atorvastatin calcium by self-emulsifying drug delivery systems (SEDDS). Pharm Dev Technol. 2011;16(1):65-74. doi: 10.3109/10837450903499333, PMID 20088679.

10. Patel JK, Patel RP, Patel M. Formulation and evaluation mucoadhesive glipizide microspheres. Vol. 24. American Association of Pharmaceutical Scientists; 2005. p. 50-5.

11. Wise LD. Handbook of pharmaceutical controlled release. First Indian reprint edition. NY: Marcel Dekker; 2005. p. 517-625.

12. Woodley J. Bioadhesion: new possibilities for drug administration? Clin Pharmacokinet. 2001;40(2):77-84. doi: 10.2165/00003088-20014002000001, PMID 11286325.

13. Dewangan HK. Albumin as natural versatile drug carrier for various diseases treatment. Agric Rev. 2020;43:239-68. doi: 10.1007/978-3-030-41838-0_8.

14. Philip AK, Philip B. Colon targeted drug delivery systems: a review on primary and novel approaches. Oman Med J. 2010;25(2):79-87. doi: 10.5001/ omj.2010.24, PMID 22125706.

15. Chowdary KP, Rao YS. Mucoadhesive microspheres for Controlled Drug Delivery. Biol Pharm Bull. 2004;27(11):1717-24. doi: 10.1248/bpb.27.1717, PMID 15516712.

16. Peng D, Huang K, Liu Y, Liu S. Preparation of novel polymeric microspheres for controlled release of finasteride. Int J Pharm. 2007;342(1-2):82-6. doi: 10.1016/j.jpharm.2007.05.002.

17. Dhaliwal S, Jain S, Singh HP, Tiwary AK. Mucoadhesive microspheres for gastroretentive delivery of acyclovir: in vitro and in vivo evaluation. AAPS J. 2008;10(2):322-30. doi: 10.1208/s12248-008-9039-2, PMID 18523891.

18. Thakral NK, Ray AR, Bar-Shalom D, Eriksson AH, Majumdar DK. The quest for targeted delivery in colon cancer: mucoadhesive valdecoxib microspheres. Int J Nanomedicine. 2011;6:1057-68. doi: 10.2147/IJN.S19561, PMID 21720517.

19. Dewangan HK, Pandey T, Maurya L, Singh S. Rational design and evaluation of $\mathrm{HBsAg}$ polymeric nanoparticles as antigen delivery carriers. Int $\mathrm{J}$ Biol Macromol. 2018;111:804-12. doi: 10.1016/j.ijbiomac.2018.01.073, PMID 29343454.

20. Deepika D, Dewangan HK, Maurya L, Singh S. Intranasal drug delivery of frovatriptan succinate-loaded polymeric nanoparticles for brain targeting. J Pharm Sci. 2019;108(2):851-9. doi: 10.1016/j.xphs.2018.07.013, PMID 30053555.
21. Umamaheshwari RB, Jain S, Bhadra D, Jain NK. Floating microspheres bearing acetohydroxamic acid for the treatment of Helicobacter pylori. J Pharm Pharmacol. 2003;55(12):1607-13. doi: 10.1211/0022357022223, PMID 14738585.

22. Khan FN, Dehghan MHG. Enhanced bioavailability and dissolution of atorvastatin calcium from floating microcapsules using minimum additives. Sci Pharm. 2012;80(1):215-28. doi: 10.3797/scipharm.1104-26, PMID 22396916.

23. Ribeir J, Silva M, Figueiredo M. Microencapsulation of hemoglobin in chitosan-coated alginate microspheres prepared by emulsification/internal gelation. Vol. 88. American Association of Pharmaceutical Scientists; 2006. p. 45-9.

24. Das MK, Senapati PC. Furosemide-loaded Alginate Microspheres Prepared by lonic Cross-linking Technique: Morphology and Release Characteristics. Indian J Pharm Sci. 2008;70(1):77-84. doi: 10.4103/0250-474X.40336, PMID 20390085.

25. Zhu X, Zhou D, Jin Y, Song YP, Zhang ZR, Huang Y. A novel microsphere with a three-layer structure for duodenum-specific drug delivery. Int J Pharm. 2011;413(1-2):110-8. doi: 10.1016/j.ijpharm.2011.04.036, PMID 21536116.

26. Dewangan HK, Singh S, Maurya L, Srivastava A. Hepatitis B antigen loaded biodegradable polymeric nanoparticles: formulation optimization and in-vivo immunization in BALB/c mice. Curr Drug Deliv. 2018;15(8):1204-15. doi: 10.2 174/1567201815666180604110457, PMID 29866006.

27. Sharma V, Dewangan HK, Maurya L, Vats K, Verma H, Singh S. Rational design and in-vivo estimation of ivabradine hydrochloride loaded nanoparticles for management of stable angina. J Drug Deliv Sci Technol. 2019;54. doi: 10.1016/j.jddst.2019.101337, PMID 101337.

28. Tao Y, Lu Y, Sun Y, Gu B, Lu W, Pan J. Development of mucoadhesive microspheres of acyclovir with enhanced bioavailability. Sun. Int J Pharm. 2009;378(1-2):30-6. doi: 10.1016/j.jpharm.2009.05.025, PMID 19465102.

29. Ranga Rao KV, Buri P. A novel in situ method to test polymers and coated microparticles for bioadhesion. I nternational. J Pharm. 1989;52:265-70.

30. Freitas MN, Marchetti JM. Nimesulide PLA microspheres as a potential sustained release system for the treatment of inflammatory diseases. Int $\mathrm{J}$ Pharm. 2005;295(1-2):201-11. doi: 10.1016/j.jpharm.2005.03.003, PMID 15848005.

31. Lakshmi, Singh S, Vijayakumar MR, Dewangan HK. Lipid based aqueous core nanocapsules (ACNs) for encapsulating hydrophillic vinorelbine bitartrate: preparation, optimization, characterization and in vitro safety assessment for intravenous Administration. Curr Drug Deliv. 2018;15(9):1284-93. doi: 10.217 $4 / 1567201815666180716112457$.

\section{SUMMARY}

Atorvastatin Calcium most effective common phytoconstituents used as lowering the cholesterol levels and primarily used for curing of dyslipidemia and cardiovascular disease. It is the competitive inhibitor of hydroxyl methylglutaryl-coenzyme A reductase, followed mevalonate pathway. One of the drawbacks of Atorvastatin Calcium have to low bioavailability and poor solubility. Current work focuses on the development of atorvastatin calcium mucoadhesive microspheres to boost delayed transit, continuous longer-term release and preclinical pharmacokinetic estimation in rabbits. By emulsification process the microsphere was prepared in which the independent variables (like polymer quantity) was studied on critical quality attributes such as entrapment efficiency. The prepare microsphere were characterized as their in-vitro release, percentage yield, physicochemical parameters, micromeritic properties, DSC, FT-IR, mucoadhesive wash off test and pharmacokinetic study in rabbit. Developed microsphere have smooth surface, negative zeta potential, maximum trapping efficiency and sustained release. Microspheres satisfy the micromeritic properties and, verified by FT-IR and DSC, showed no interaction between drug and polymer. The in-vivo study showed that the prepared microspheres are efficient for longer-lasting colon-targeted drug delivery systems. Relatively steady plasma drug concentrations were observed in the pharmacokinetics analysis after oral drug administration. For the formulation, $\mathrm{AUCO}_{-24 \mathrm{~h}}$ was significantly higher than for the pure drug. The preclinical oral bioavailability analysis of the drug was improved as relative availability values were high compared to the pure drug and transit time was delayed for longer periods of time. 
PICTORIAL ABSTRACT

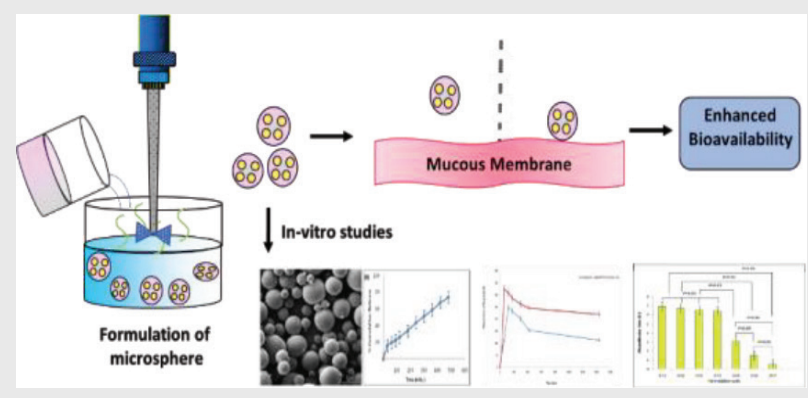

About Authors

Dr. Hitesh Kumar Dewangan: Ph.D., Pharmaceutics; Assistant Professor at Institute of Pharmaceutical Research, GLA University, NH-2 Mathura Delhi Road, P.O.- Chaumuhan, Mathura. Research Interest: Nanotechnology, drug delivery, Pharmaceutics.

Mrs. Arpana Sharma: M. Pharm, Pharmaceutics; Faculty of Pharmacy, VNS Group of Institution, Bhopal, Madhya Pradesh, India. Research Interest: Drug delivery, Pharmaceutics.

Dr. Ankit Mishra: Ph.D., Pharmaceutics; Assistant Professor at Faculty of Pharmacy, VNS Group of Institution, Bhopal, Madhya Pradesh, India. Research Interest: Drug delivery, Pharmaceutics.

Dr. Pradeep Singour: Ph.D., Medicinal Chemistry; Associate Professor, Faculty of Pharmacy, VNS Group of Institution, Bhopal, Madhya Pradesh, India. Research Interest: Drug delivery, Molecular synthesis.

Cite this article: Dewangan HK, Sharma A, Mishra A, Singour P. Mucoadhesive Microspheres of Atorvastatin Calcium: Rational Design, Evaluation and Enhancement of Bioavailability. Indian $\mathrm{J}$ of Pharmaceutical Education and Research. 2021;55(3s):s733-s741. 